\title{
Fault Reconstruction Approach for Distributed Coordinated Spacecraft Attitude Control System
}

\author{
Mingyi Huo, ${ }^{1}$ Yanning Guo, ${ }^{1}$ and Xing Huo ${ }^{2}$ \\ ${ }^{1}$ Department of Control Science and Engineering, Harbin Institute of Technology, Harbin 150001, China \\ ${ }^{2}$ College of Engineering, Bohai University, Jinzhou 121000, China \\ Correspondence should be addressed to Xing Huo; hmyi888@163.com
}

Received 15 January 2015; Accepted 2 March 2015

Academic Editor: Kun Liu

Copyright (c) 2015 Mingyi Huo et al. This is an open access article distributed under the Creative Commons Attribution License, which permits unrestricted use, distribution, and reproduction in any medium, provided the original work is properly cited.

\begin{abstract}
This work presents a novel fault reconstruction approach for a large-scale system, that is, a distributed coordinated spacecraft attitude control system. The attitude of all the spacecrafts in this distributed system is controlled by using thrusters. All possible faults of thruster including thrust magnitude error and alignment error are investigated. As a stepping stone, the mathematical model of thruster is firstly established based on the thruster configuration. On the basis of this, a sliding mode observer is then proposed to reconstruct faults in each agent of the coordinated control system. A Lyapunov-based analysis shows that the observer asymptotically converges to the actual faults. The key feature of this fault reconstruction approach is that it can achieve a faster reconstruction of the fault in comparison with the conventional fault reconstruction schemes. It can globally reconstruct thruster faults with zero reconstruction error, and this is accomplished within finite time. The effectiveness of the proposed approach is analytically authenticated via simulation study.
\end{abstract}

\section{Introduction}

Distributed coordinate spacecraft attitude control system, as a large-scale system, is revolutionizing our way for performing space missions. This brings on several advantages in space mission accomplishment. It usually includes two or more spacecrafts in formation flying. It is to distribute the functionality of a single large/complex spacecraft to a set of smaller, less-expensive cooperative spacecrafts. In recent years, NASA, the U.S. Air Force, and the ESA have shown keen interests in developing reliable autonomous formation strategies to deploy multiple spacecrafts for various space missions [1]. The advantages of multiple-spacecraft formation flying include increasing resolution of scientific observations, reducing cost, enhancing overall system robustness, and adding flexibility to space-based programs. However, it also poses tremendous challenges, such as spacecraft formation initialization to reconfiguration, coordination, and formation trajectory generation. More specifically, distributed coordinate spacecraft attitude control is still an open problem that needs to be further solved.
In the past decades, the problem of distributed coordinated attitude control for spacecraft formation flying has been intensively investigated [2-4]. Classically, the leaderfollower [5], the behavioral based approach [6,7], and the virtual structure [8] are three schemes for the spacecraft formation synchronization. In [9], coordinated control for multiple spacecraft was discussed. In [8], synchronization of spacecraft formation was investigated by using virtual structure technique. Parametric uncertainties and external disturbance were also addressed. In [10], a robust distributed coordinated attitude control law was presented by using behavioral based approach. More specifically, due to its fast convergence rate and its superiority of stabilizing the system within finite time, finite-time controller design for distributed coordinate spacecraft attitude system has attracted more and more attention in recent years. In [11], finite-time attitude synchronization and stabilization problem were investigated for spacecrafts. The designed controller was able to guarantee the finite-time stability of the closed-loop system.

Most of the previous research, however, handles distributed coordinate spacecraft attitude control based on 
the assumption that an exact model of the actuator is available. This assumption is rarely satisfied in practice because the actuator parameters may have uncertainties due to installation error, aging and wearing out of the mechanical and electrical parts, and so forth. The first type of uncertainty in actuator that needs to be tackled is actuator faults [12]. Once a spacecraft is launched, it is highly unlikely that its hardware can be repaired. Thus any component or system failure cannot be fixed with replacement parts. These issues can potentially cause a host of economic, environmental, and safety problems. SMC with actuator faults does not seem to have received much attention in the literature. In [13], a SMC based controller was proposed to achieve reliable attitude stabilization, while actuator outage faults were accommodated. An adaptive SMC control approach was proposed in [14] for performing attitude tracking maneuvers in the presence of disturbances and thruster's failures. Another adaptive SMC attitude control was presented in [15] to handle several fault scenarios of rotating solar flaps. The authors in [16] looked at a terminal sliding mode control approach for the satellite formations flying. However, stability analysis of the closedloop system was not provided when the faults occurred. For a flexible spacecraft with partial loss of control effectiveness fault, a SMC control was developed in [17] to accomplish attitude tracking.

Active FTC relies on the availability of a Fault Detection and Isolation (FDI) block that detects and identifies fault online [18-21] and then reacts to the system fault actively by reconfiguring controller. The application of active FTC to satellite attitude control, especially the FDI design, has attracted considerable interests. A dynamic neural network scheme was presented in [22] to detect and isolate reaction wheel faults. In [23], the problem of detecting reaction wheel faults in a tetrahedron configuration was investigated. An iterative learning observer-based FDI was reported in [24] to estimate time-varying thruster faults. In [25], a twostage Kalman filtering algorithm was developed to estimate reaction wheel faults; a fault-tolerant controller was then synthesized to accommodate the faults. Two model-based schemes were developed in [26] by using $\mathscr{H}_{\mathrm{\infty}} / \mathscr{H}_{2}$ filters to address the fault diagnosis problem of micro thrusters. In [27], a set of fault detection filters were presented for deep space satellites to detect and identify faults in sensors or actuators. In [28], the problem of robust FDI design for thruster faults in the Mars Express satellite was discussed. For a benchmark Mars Express satellite, the authors in $[29,30]$ presented a nonlinear sliding mode observer to identify and isolate faults induced in thruster and sensors.

Another type of uncertainty in actuator that should be addressed is actuator misalignments. Due to finitemanufacturing tolerances or warping of the spacecraft structure during launch, some actuator alignment error will definitely exist. That problem may cause the onboard control algorithm to fail and thus pose significant risk to the successful operation of the spacecraft. It is thus desirable to design a control methodology to handle actuator misalignments. Unfortunately, there has been insufficient research on control in the presence of actuator misalignments. One paper developed an adaptive control law to accomplish attitude maneuver in the presence of relatively small gimbals' alignment error of variable speed control moment gyros [31]. In [32], a nonlinear model reference adaptive control scheme was tested in the presence of alignment errors up to fifteen degrees. Although an extended Kalman filter was used in another approach to develop methods for on-orbit actuator alignment calibration, uncertain inertia properties were not taken into account [33]. In another study [34], an adaptive control approach was proposed for satellite formation flying. The backstepping technique was used to synthesize the controller, and the thrust magnitude error and misalignment were successfully handled.

Based on the above analysis, it is known that if the fault occurring in any spacecraft can not be successfully in realtime, then it may degrade the whole performance of the distributed coordinate spacecraft attitude control system. Sometimes, it would fail the space missions of the spacecraft formation flying. This issue may pose a question for us; that is, if the fault in each spacecraft can be exactly diagnosed or reconstructed, then a fault-tolerant coordinate attitude controller can be designed as follows to guarantee the acceptable performance even in the presence of fault. Motivated by this, this study will investigate the problem of fault reconstruction for each spacecraft of the considered coordinate spacecraft system. In this work, a sliding mode observer-based reconstruction approach will be presented to estimate the faults. This approach can achieve a faster reconstruction in comparison with the conventional fault diagnosis schemes. Moreover, it is able to estimate the occurred fault in finite time with zero reconstruction error.

The remainder of this paper is organized as follows: In Section 2, problem formulation is presented including mathematical model and problem statement. A sliding mode observer-based fault reconstruction approach is developed in Section 3, and also the stability of closed-loop observer error system is provided. In Section 4, simulation results with the application of the designed fault reconstruction scheme to a distributed coordinated spacecraft attitude control system are presented. Section 5 presents some concluding remarks and future work.

\section{Problem Formulation}

The notation adopted throughout this paper is introduced as follows: Let $\mathbf{I}_{n} \in \mathfrak{R}^{n \times n}$ denote the $n$-by- $n$ identity matrix and the symbol $\|\cdot\|$ denotes the Euclidean norm or its induced norm. For vector $\mathbf{x}=\left[\begin{array}{llll}x_{1} & x_{2} & \cdots & x_{n}\end{array}\right]^{\mathrm{T}}$ $\epsilon \mathfrak{R}^{n}$, a vector function is defined as $\operatorname{sgn}(\mathbf{x})=$ $\left[\begin{array}{llll}\operatorname{sign}\left(x_{1}\right) & \operatorname{sign}\left(x_{2}\right) & \cdots & \operatorname{sign}\left(x_{n}\right)\end{array}\right]^{\mathrm{T}}$ with $\operatorname{sign}(\cdot)$ the sign function.

2.1. Mathematical Model of Distributed Coordinated Spacecraft Attitude Control System. In this work, each spacecraft in the distributed coordinated system is modeled as a rigid body in a three-dimensional space, and an external disturbance is not considered in the model description. For a distributed coordinate system with $n$ spacecrafts, let $\mathscr{F}_{I}$ denote the inertial frame and $\mathscr{F}_{b}^{i}$ denote the body-fixed frame of the $i$ th 
spacecraft. For each spacecraft, given a Euler rotation angle $\phi_{i}(t) \in \mathfrak{R}$ about the Euler principle axis $\mathbf{n}_{i} \in \mathfrak{R}^{3}$, the attitude orientation of the $i$ th spacecraft in $\mathscr{F}_{b}^{i}$ with respect to $\mathscr{F}_{I}$ can be represented by the MRP vector $\boldsymbol{\sigma}_{b}^{i}=\left[\begin{array}{lll}\sigma_{1}^{i} & \sigma_{2}^{i} & \sigma_{3}^{i}\end{array}\right]^{\mathrm{T}} \in \mathfrak{R}^{3}$, which is given by [35] $\boldsymbol{\sigma}_{b}^{i}=\mathbf{n}_{i} \tan \left(\phi_{i}(t) / 4\right), \phi_{i}(t) \in\left[0^{\circ}, 360^{\circ}\right)$.

Let the angular velocity of the $i$ th spacecraft with respect to the inertial reference frame $\mathscr{F}_{I}$ and expressed in the bodyfixed frame $\mathscr{F}_{b}^{i}$ be denoted by $\boldsymbol{\omega}_{b}^{i} \in \mathfrak{R}^{3}$. Using the MRPs, the kinematic differential equation of the $i$ th spacecraft can be determined as follows [35]:

$$
\begin{aligned}
\dot{\boldsymbol{\sigma}}_{b}^{i} & =\frac{1}{4}\left[\left(1-\left(\boldsymbol{\sigma}_{b}^{i}\right)^{\mathrm{T}} \boldsymbol{\sigma}_{b}^{i}\right) \mathbf{I}_{3}+2 \mathbf{S}\left(\boldsymbol{\sigma}_{b}^{i}\right)+2 \boldsymbol{\sigma}_{b}^{i}\left(\boldsymbol{\sigma}_{b}^{i}\right)^{\mathrm{T}}\right] \boldsymbol{\omega}_{b}^{i} \\
& =\mathbf{G}_{i}\left(\boldsymbol{\sigma}_{b}^{i}\right) \boldsymbol{\omega}_{b}^{i},
\end{aligned}
$$

where the matrix $\mathbf{S}(\mathbf{x})$ is the skew-symmetric matrix such that $\mathbf{S}(\mathbf{x}) \mathbf{y}=\mathbf{x} \otimes \mathbf{y}$ for any vectors $\mathbf{x}, \mathbf{y} \in \mathfrak{R}^{3}$, with " $\otimes$ " being the vector cross product.

The dynamical model of the $i$ th spacecraft motion can be found from Euler's moment equation, and it is given by [36]

$$
\mathbf{J}_{i} \dot{\boldsymbol{\omega}}_{b}^{i}=-\mathbf{S}\left(\boldsymbol{\omega}_{b}^{i}\right) \mathbf{J}_{i} \boldsymbol{\omega}_{b}^{i}+\mathbf{u}_{i},
$$

where $\mathbf{J}_{i} \in \mathfrak{R}^{3 \times 3}$ (positive and definite) is the total inertia matrix of the $i$ th spacecraft and $\mathbf{u}_{i}=\left[\begin{array}{lll}u_{1}^{i} & u_{2}^{i} & u_{3}^{i}\end{array}\right]^{\mathrm{T}} \in \mathfrak{R}^{3}$ denotes the total control torque generated by all the mounted actuators.

In this work, information exchange among agents can be represented as a graph. Graph $\mathscr{G}$ consists of a node set $\mathscr{V}=\{1,2, \ldots, n\}$, an edge set $\mathscr{E} \subseteq \mathscr{V} \times \mathscr{V}$, and a weighted adjacency matrix $\Lambda=\left[a_{j n}\right] \in \mathfrak{R}^{n \times n}$. To define the communication topology in the network we consider $n$ agent spacecraft as nodes of a graph, called communication graph. The communication links among the agents are considered as the communication graph edge set.

2.2. Thruster Fault of Each Spacecraft. Because thruster can generate larger control torque than reaction wheels, it becomes one type of actuators commonly used in large-angle attitude maneuver. Thus, all the agents in the distributed coordinated spacecraft system considered in this work are controlled by using thrusters. A thruster consists of a flow control valve and a combustion chamber. When propellant passes through the combustion chamber, chemical reaction takes place generating thrust through the nozzle.

Assume that $N_{i}$ thrusters are mounted in the $i$ th spacecraft. For the $j$ th thruster in the $i$ th spacecraft, $j=1,2, \ldots, N_{i}$, its configuration is shown in Figure 2; the force component can be derived as

$$
\mathbf{F}_{i j}=F\left[\begin{array}{c}
\cos \alpha_{i j} \cos \beta_{i j} \\
\cos \alpha_{i j} \sin \beta_{i j} \\
\sin \alpha_{i j}
\end{array}\right],
$$

where $F>0$ is the constant thrust level, $\alpha_{i j}$ is the elevation angle, and $\beta_{i j}$ is the azimuth angle. Let $\mathbf{r}_{i j}=r_{x i j} \mathbf{X}_{B i}+$ $r_{y i j} \mathbf{Y}_{B i}+r_{z i j} \mathbf{Z}_{B i}$ be the vector representing the placement of the reaction thruster from the satellite center of mass. Torque component provided by the $j$ th thruster can be calculated as

$$
\boldsymbol{\tau}_{i j}=\mathbf{r}_{i j} \times \mathbf{F}_{i j} .
$$

Then, the applied control torque $\mathbf{u}_{i}$ generated by $N_{i}$ thrusters is

$$
\mathbf{u}_{i}=\sum_{j=1}^{N_{i}} \boldsymbol{\tau}_{i j}=\sum_{j=1}^{N_{i}} \mathbf{r}_{i j} \times \mathbf{F}_{i j} .
$$

In this study, thruster faults including misalignment error and thrust magnitude error are considered. The nature of those two scenarios is described as follows.

(1) Misalignment Error. In practical aerospace engineering, the configuration of actuators is not perfect. Misalignment error may exist due to space debris or finite-manufacturing technique. As a result, the demanded torque from controller is different from the torque produced by the actuators. For the $j$ th thruster in the $i$ th spacecraft, misalignment error may exist in $\mathbf{r}_{i j}$ and the alignment angles $\alpha_{i j}, \beta_{i j}$. Let $\mathbf{r}_{i j}^{0}, \Delta \mathbf{r}_{i j}$ denote the nominal and the alignment error distance between satellite center and the thruster, respectively. Then, $\mathbf{r}_{i j}$ can be rewritten as $\mathbf{r}_{i j}=\mathbf{r}_{i j}^{0}+\Delta \mathbf{r}_{i j}$. Assume that the thruster is tilted over nominal direction with small constant angles, $\Delta \alpha_{i j}$ and $\Delta \beta_{i j}$. Then, the alignment angle can be denoted as $\alpha_{i j}=\alpha_{i j}^{0}+\Delta \alpha_{i j}$ and $\beta_{i j}=\beta_{i j}^{0}+\Delta \beta_{i j}$, where $\alpha_{i j}^{0}$ and $\beta_{i j}^{0}$ are the nominal alignment angle.

(2) Thrust Magnitude Error. As discussed in [37], due to reduction in the amount of propellant's mass, the amount of thrust generated is reduced. Moreover, due to wear and tear, the conductivity of the wires, capacitor, and electrodes may decrease. Consequently, the amount of discharge current produced during the generation of pulses is reduced, resulting in the reduction of the amount of thrust produced. Those two issues will inevitably introduce the problem of thrust magnitude error for thruster. For the $j$ th thruster in the $i$ th spacecraft, let $F_{0}$ and $\Delta F_{i j}$ represent the nominal and error of thrust magnitude, respectively. The actual thrust $F$ can thus be denoted by

$$
F=F_{0}+\Delta F_{i j} .
$$

Taking thruster magnitude error and alignment error into consideration, $\boldsymbol{\tau}_{i j}$ in (4) can be rewritten as

$$
\begin{aligned}
\boldsymbol{\tau}_{i j}= & \left(F_{0}+\Delta F_{i j}\right)\left(\mathbf{r}_{i j}^{0}+\Delta \mathbf{r}_{i j}\right) \\
& \times\left[\begin{array}{c}
\cos \left(\alpha_{i j}^{0}+\Delta \alpha_{i j}\right) \cos \left(\beta_{i j}^{0}+\Delta \beta_{i j}\right) \\
\cos \left(\alpha_{i j}^{0}+\Delta \alpha_{i j}\right) \sin \left(\beta_{i j}^{0}+\Delta \beta_{i j}\right) \\
\sin \left(\alpha_{i j}^{0}+\Delta \alpha_{i j}\right)
\end{array}\right] \\
= & F_{0}\left(\mathbf{r}_{i j}^{0}\right) \times \mathbf{D}_{i j}^{0}+\Delta F\left(\mathbf{r}_{i j}^{0}\right) \times \mathbf{D}_{i j}^{0} \\
& +\left(F_{0}+\Delta F_{i j}\right)\left[\left(\mathbf{r}_{i j}^{0}\right) \times \Delta \mathbf{D}_{i j}+\Delta \mathbf{r}_{i j} \times\left(\mathbf{D}_{i j}^{0}+\Delta \mathbf{D}_{i j}\right)\right],
\end{aligned}
$$


where

$$
\begin{aligned}
\mathbf{D}_{i j}^{0}= & {\left[\begin{array}{c}
\cos \alpha_{i j}^{0} \cos \beta_{i j}^{0} \\
\cos \alpha_{i j}^{0} \sin \beta_{i j}^{0} \\
\sin \alpha_{i j}^{0}
\end{array}\right], } \\
\Delta \mathbf{D}_{i j}= & {\left[\begin{array}{c}
\cos \left(\alpha_{i j}^{0}+\Delta \alpha_{i j}\right) \cos \left(\beta_{i j}^{0}+\Delta \beta_{i j}\right) \\
\cos \left(\alpha_{i j}^{0}+\Delta \alpha_{i j}\right) \sin \left(\beta_{i j}^{0}+\Delta \beta_{i j}\right) \\
\sin \left(\alpha_{i j}^{0}+\Delta \alpha_{i j}\right)
\end{array}\right] } \\
& -\left[\begin{array}{c}
\cos \alpha_{i j}^{0} \cos \beta_{i j}^{0} \\
\cos \alpha_{i j}^{0} \sin \beta_{i j}^{0} \\
\sin \alpha_{i j}^{0}
\end{array}\right] .
\end{aligned}
$$

From (5) and (7), the real/total thrust force with magnitude error and misalignment is expressed as the sum of nominal and thrust error terms in the body-fixed frame:

$\mathbf{u}_{i}$

$$
\begin{aligned}
= & F_{0}^{F_{j=1}^{N_{i}}\left(\mathbf{r}_{i j}^{0}\right) \times \mathbf{D}_{i j}^{0}} \\
& +\underbrace{\sum_{j=1}^{N_{i}}\left\{\Delta F_{i j}\left(\mathbf{r}_{i j}^{0}\right) \times \mathbf{D}_{i j}^{0}+\left(F_{0}+\Delta F_{i j}\right)\left[\left(\mathbf{r}_{i j}^{0}\right) \times \Delta \mathbf{D}_{i j}+\Delta \mathbf{r}_{i j} \times\left(\mathbf{D}_{i j}^{0}+\Delta \mathbf{D}_{i j}\right)\right]\right\}}_{\mathbf{u}_{\text {nor } j}},
\end{aligned}
$$

where the vector $\mathbf{u}_{\text {nor } i} \in \mathfrak{R}^{3}$ is the nominal control torque commanded by the controller of the $i$ th spacecraft and $\mathbf{u}_{\text {fault } i} \in \mathfrak{R}^{3}$ denotes the faulty torque induced by misalignment error and thrust magnitude error of thrusters in the $i$ th spacecraft.

2.3. Problem Statement. In this study, our objective to be achieved can be stated as follows: Consider the distributed coordinate spacecraft attitude control system and design an observer-based reconstruction approach to reconstruct all possible faults occurring in thrusters of all the distributed coordinate system. Moreover, the reconstruction error of the faults should be governed to zero in finite time, and a faster reconstruction of thruster fault should be accomplished.

\section{Sliding Mode Observer-Based Fault Reconstruction Approach Design}

A sliding mode observer-based fault reconstruction approach will be developed for the distributed coordinate system. With application of this approach, the thruster fault occurring in each spacecraft will be reconstructed. The reconstruction error will converge to zero with finite-time convergence. In this section, the upper bound of the thruster faults will be firstly analyzed or estimated. This estimated upper bound will be useful for the choice of the observer gains in the following proposed sliding mode observer.

3.1. Analysis of the Upper Bound of the Thruster Fault. Define a matrix $\mathbf{T}_{i}\left(\boldsymbol{\sigma}_{b}^{i}\right)=\left(\mathbf{G}_{i}\left(\boldsymbol{\sigma}_{b}^{i}\right)\right)^{-1}$; combining with the spacecraft kinematics (1) and its dynamics (2), taking thruster fault (10) into consideration, it can establish the following transformed two-order differential equation:

$$
\begin{aligned}
\overline{\mathbf{J}}_{i}\left(\boldsymbol{\sigma}_{b}^{i}\right) \ddot{\boldsymbol{\sigma}}_{b}^{i}+\mathbf{C}_{i}\left(\boldsymbol{\sigma}_{b}^{i}, \dot{\boldsymbol{\sigma}}_{b}^{i}\right) \dot{\boldsymbol{\sigma}}_{b}^{i} \\
\quad=\left(\mathbf{T}_{i}\left(\boldsymbol{\sigma}_{b}^{i}\right)\right)^{\mathrm{T}} \mathbf{u}_{\mathrm{nor} i}+\left(\mathbf{T}_{i}\left(\boldsymbol{\sigma}_{b}^{i}\right)\right)^{\mathrm{T}} \mathbf{u}_{\mathrm{fault} i},
\end{aligned}
$$

where $\quad \mathbf{C}_{i}\left(\boldsymbol{\sigma}_{b}^{i}, \dot{\boldsymbol{\sigma}}_{b}^{i}\right) \quad=\quad\left(\mathbf{T}_{i}\left(\boldsymbol{\sigma}_{b}^{i}\right)\right)^{\mathrm{T}} \mathbf{J}_{i} \dot{\mathbf{T}}_{i}\left(\boldsymbol{\sigma}_{b}^{i}\right)+$ $\left(\mathbf{T}_{i}\left(\boldsymbol{\sigma}_{b}^{i}\right)\right)^{\mathrm{T}} \mathbf{S}\left(\mathbf{T}_{i}\left(\boldsymbol{\sigma}_{b}^{i}\right) \dot{\boldsymbol{\sigma}}_{b}^{i}\right) \mathbf{J}_{i} \mathbf{T}_{i}\left(\boldsymbol{\sigma}_{b}^{i}\right), \overline{\mathbf{J}}_{i}=\left(\mathbf{T}_{i}\left(\boldsymbol{\sigma}_{b}^{i}\right)\right)^{\mathrm{T}} \mathbf{J}_{i} \mathbf{T}_{i}\left(\boldsymbol{\sigma}_{b}^{i}\right)$.

Property 1. The matrix $\mathbf{G}_{i}\left(\boldsymbol{\sigma}_{b}^{i}\right)$ is such that [38]

$$
\begin{gathered}
\mathbf{T}_{i}\left(\boldsymbol{\sigma}_{b}^{i}\right)=\frac{16}{\left(1+\left(\boldsymbol{\sigma}_{b}^{i}\right)^{\mathrm{T}} \boldsymbol{\sigma}_{b}^{i}\right)^{2}}\left(\mathbf{G}_{i}\left(\boldsymbol{\sigma}_{b}^{i}\right)\right)^{\mathrm{T}} \\
\left(\mathbf{G}_{i}\left(\boldsymbol{\sigma}_{b}^{i}\right)\right)^{\mathrm{T}} \mathbf{G}_{i}\left(\boldsymbol{\sigma}_{b}^{i}\right)=\left(\frac{1+\left(\boldsymbol{\sigma}_{b}^{i}\right)^{\mathrm{T}} \boldsymbol{\sigma}_{b}^{i}}{4}\right)^{2} \mathbf{I}_{3} .
\end{gathered}
$$

Property 2. The matrix $\overline{\mathbf{J}}_{i}\left(\boldsymbol{\sigma}_{b}^{i}\right)$ is positive-definite and symmetric. There exist two positive scalars $\lambda_{\min i} \in \Re$ and $\lambda_{\max i} \in \Re$ such that $\lambda_{\min i}\|\mathbf{x}\|^{2} \leq \mathbf{x}^{\mathrm{T}} \overline{\mathbf{J}}_{i}\left(\boldsymbol{\sigma}_{b}^{i}\right) \mathbf{x} \leq \lambda_{\max i}\|\mathbf{x}\|^{2}$ for any vector $\mathbf{x} \in \mathfrak{R}^{3}$.

Property 3. The matrix $\mathbf{C}_{i}\left(\boldsymbol{\sigma}_{b}^{i}, \dot{\boldsymbol{\sigma}}_{b}^{i}\right)$ and the time-derivative of $\overline{\mathbf{J}}_{i}\left(\boldsymbol{\sigma}_{b}^{i}\right)$ satisfy the skew-symmetric relationship [38] $\mathbf{x}^{\mathrm{T}}\left(\dot{\overline{\mathbf{J}}}_{i}\left(\boldsymbol{\sigma}_{b}^{i}\right)-2 \mathbf{C}_{i}\left(\boldsymbol{\sigma}_{b}^{i}, \dot{\boldsymbol{\sigma}}_{b}^{i}\right)\right) \mathbf{x}=0$ for all $\mathbf{x} \in \boldsymbol{R}^{3}$.

Theorem 1. For the thruster fault $\mathbf{u}_{\text {fault_i }}$ in (10), it is bounded by a positive and known constant $\gamma_{i} \in \mathfrak{R}$. Moreover, if the term $\left(\mathbf{T}_{i}\left(\boldsymbol{\sigma}_{b}^{i}\right)\right)^{\mathrm{T}} \mathbf{u}_{\text {fault } i}$ in (11) is viewed as a lumped fault, then this can be bounded by a positive constant $8 \gamma_{i}$; that is, $\left\|\left(\mathbf{T}_{i}\left(\boldsymbol{\sigma}_{b}^{i}\right)\right)^{\mathrm{T}} \mathbf{u}_{\text {fault } i}\right\| \leq 8 \gamma_{i}$.

Proof. As shown in (10), it is known that

$$
\begin{aligned}
\mathbf{u}_{\text {fault } i}=\sum_{j=1}^{N_{i}}\left\{\Delta F_{i j}\left(\mathbf{r}_{i j}^{0}\right) \times \mathbf{D}_{i j}^{0}+\left(F_{0}+\Delta F_{i j}\right)\right. \\
\left.\cdot\left[\left(\mathbf{r}_{i j}^{0}\right) \times \Delta \mathbf{D}_{i j}+\Delta \mathbf{r}_{i j} \times\left(\mathbf{D}_{i j}^{0}+\Delta \mathbf{D}_{i j}\right)\right]\right\} .
\end{aligned}
$$

According to the physical limitation of thruster, the thrust magnitude error should absolutely satisfy

$$
\left|\Delta F_{i j}\right| \leq F
$$

On the other hand, although there exists alignment error in each thruster of the $i$ th spacecraft, this misalignment should be finite, and it will be always such that

$$
\left\|\Delta \mathbf{r}_{i j}\right\| \leq\left\|\mathbf{r}_{i}^{0}\right\|
$$


From (14) and (15), the following inequality can be obtained:

$$
\begin{aligned}
&\left\|\mathbf{u}_{\text {fault } i \text { i }}\right\| \leq \sum_{j=1}^{N_{i}} \| \Delta F_{i j}\left(\mathbf{r}_{i j}^{0}\right) \times \mathbf{D}_{i j}^{0}+\left(F_{0}+\Delta F_{i j}\right) \cdot\left[\left(\mathbf{r}_{i j}^{0}\right) \times \Delta \mathbf{D}_{i j}+\Delta \mathbf{r}_{i j} \times\left(\mathbf{D}_{i j}^{0}+\Delta \mathbf{D}_{i j}\right)\right] \| \\
& \leq F \sum_{j=1}^{N_{i}}\left\{\left\|\left(\mathbf{r}_{i j}^{0}\right) \times \mathbf{D}_{i j}^{0}\right\|\right. \\
&\left.+2\left[\left\|\left(\mathbf{r}_{i j}^{0}\right) \times \Delta \mathbf{D}_{i j}+\Delta \mathbf{r}_{i j} \times\left(\mathbf{D}_{i j}^{0}+\Delta \mathbf{D}_{i j}\right)\right\|\right]\right\} .
\end{aligned}
$$

With the definition $\Delta \mathbf{D}_{i j}$ and $\mathbf{D}_{i j}^{0}$ in (8) and (9), respectively, it follows that $\left\|\Delta \mathbf{D}_{i j}\right\| \leq 2 \sqrt{3}$ and $\left\|\mathbf{D}_{i j}^{0}\right\| \leq \sqrt{3}$. Then, it leaves inequality (16) as

$$
\begin{aligned}
\left\|\mathbf{u}_{\text {fault_i }}\right\| \leq & F \sum_{j=1}^{N_{i}}\left\{\left\|\left(\mathbf{r}_{i j}^{0}\right) \times \mathbf{D}_{i j}^{0}\right\|\right. \\
& \left.+2\left[\left\|\left(\mathbf{r}_{i j}^{0}\right) \times \mathbf{D}_{i j}^{0}+\Delta \mathbf{r}_{i j} \times\left(\mathbf{D}_{i j}^{0}+\Delta \mathbf{D}_{i j}\right)\right\|\right]\right\} \\
\leq & F \sum_{j=1}^{N_{i}}\left\{\sqrt{3}\left\|\mathbf{r}_{i j}^{0}\right\|+2\left[2 \sqrt{3}\left\|\mathbf{r}_{i j}^{0}\right\|+3 \sqrt{3}\left\|\mathbf{r}_{i j}^{0}\right\|\right]\right\} \\
= & 11 \sqrt{3} F \sum_{j=1}^{N_{i}}\left\|\mathbf{r}_{i j}^{0}\right\| .
\end{aligned}
$$

It can be concluded from (17) that the possible thruster fault $\mathbf{u}_{\text {fault_i } i}$ is bounded by $\gamma_{i} \leq 11 \sqrt{3} F \sum_{j=1}^{N_{i}}\left\|\mathbf{r}_{i j}^{0}\right\|$; that is, $\left\|\mathbf{u}_{\text {fault } i}\right\| \leq$ $\gamma_{i}$.

In addition, it can be obtained from (12) in Property 1 that

$$
\begin{aligned}
\left\|\mathbf{T}_{i}\left(\boldsymbol{\sigma}_{b}^{i}\right)\right\| & \leq \frac{16}{\left(1+\left(\boldsymbol{\sigma}_{b}^{i}\right)^{\mathrm{T}} \boldsymbol{\sigma}_{b}^{i}\right)^{2}}\|\|\left(\mathbf{G}_{i}\left(\boldsymbol{\sigma}_{b}^{i}\right)\right) \| \\
& \leq 16\left\|\left(\mathbf{G}_{i}\left(\boldsymbol{\sigma}_{b}^{i}\right)\right)\right\| \leq 8 .
\end{aligned}
$$

Consequently, using inequalities (17) and (18), it yields that the lumped fault $\left(\mathbf{T}_{i}\left(\boldsymbol{\sigma}_{b}^{i}\right)\right)^{\mathrm{T}} \mathbf{u}_{\text {fault_i}}$ in (11) is bounded by

$$
\left\|\left(\mathbf{T}_{i}\left(\boldsymbol{\sigma}_{b}^{i}\right)\right)^{\mathrm{T}} \mathbf{u}_{\text {fault } \_}\right\| \leq\left\|\left(\mathbf{T}_{i}\left(\boldsymbol{\sigma}_{b}^{i}\right)\right)^{\mathrm{T}}\right\|\left\|\mathbf{u}_{\text {fault } i}\right\| \leq 8 \gamma_{i} .
$$

To this end, the upper bounds of the thruster fault $\mathbf{u}_{\text {fault } i}$ and the lumped fault $\left(\mathbf{T}_{i}\left(\boldsymbol{\sigma}_{b}^{i}\right)\right)^{\mathrm{T}} \mathbf{u}_{\text {fault } i}$ are thus successfully analyzed. Thereby, the proof is completed here.
3.2. Fault Reconstruction Approach Design. For the transformed attitude dynamics (11), the following change of coordinates will be firstly introduced:

$$
\begin{aligned}
& \mathbf{x}_{i \_1}=\boldsymbol{\sigma}_{b}^{i}, \\
& \mathbf{x}_{i_{-2}}=\dot{\boldsymbol{\sigma}}_{b}^{i}, \\
& \mathbf{y}_{i_{-1}}=\left(\mathbf{T}_{i}\left(\boldsymbol{\sigma}_{b}^{i}\right)\right)^{\mathrm{T}} \mathbf{u}_{\text {fault } i} .
\end{aligned}
$$

Then, it can rewrite (11) as the following nonlinear system:

$$
\begin{aligned}
& \dot{\mathbf{x}}_{i_{-} 1}=\mathbf{x}_{i_{-} 2}, \\
& \overline{\mathbf{J}}_{i}\left(\mathbf{x}_{i \_1}\right) \dot{\mathbf{x}}_{i \_2}=-\mathbf{C}_{i}\left(\mathbf{x}_{i_{-1}}, \mathbf{x}_{i \_2}\right) \mathbf{x}_{i \_2}+\left(\mathbf{T}_{i}\left(\boldsymbol{\sigma}_{b}^{i}\right)\right)^{\mathrm{T}} \mathbf{u}_{\text {nor } \_i}+\mathbf{y}_{i_{-} 1} \text {. }
\end{aligned}
$$

Before presenting the details of the sliding mode observer-based fault reconstruction approach, the following lemma is firstly presented; it is useful for proving the stability of the proposed observer.

Lemma 2 (see [39]). Suppose that there exists a continuous positive-definite function $V_{1}(t)$ satisfying the following inequality:

$$
\dot{V}_{1}(t)+\alpha_{1} V_{1}(t)+\delta_{1} V_{1}^{\kappa}(t) \leq 0, \quad \forall t \geq 0 .
$$

Then, $V_{1}(t)$ will converge to zero in a finite-time $t_{f} \in \mathfrak{R}$,

$$
t_{f} \leq \frac{1}{\alpha_{1}(1+\kappa)} \ln \frac{\alpha_{1} V_{1}^{1-\kappa}(0)+\delta_{1}}{\delta_{1}},
$$

where $\alpha_{1}>0, \delta_{1}>0$, and $0<\kappa<1$ are scalars.

To accomplish the reconstruction of thruster fault in finite time, a sliding mode observer will be developed. The sliding surface is given as follows for each spacecraft:

$$
\mathbf{M}_{i}=\mathbf{x}_{i 2}-\boldsymbol{\xi}_{i} .
$$

In (27), $\xi_{i}$ is designed as follows:

$$
\begin{aligned}
\dot{\boldsymbol{\xi}}_{i}=\overline{\mathbf{J}}_{i}^{-1}\left(\mathbf{x}_{i \_}\right)[ & K_{i} \mathbf{M}_{i}+\mu_{i} \operatorname{sgn}\left(\mathbf{M}_{i}\right)+\varepsilon_{i} \mathbf{M}_{i}^{\pi_{i} / \vartheta_{i}} \\
& \left.+\left(\mathbf{T}_{i}\left(\boldsymbol{\sigma}_{b}^{i}\right)\right)^{\mathrm{T}} \mathbf{u}_{\text {nor } \_i}-\mathbf{C}_{i}\left(\mathbf{x}_{i_{-} 1}, \mathbf{x}_{i_{-}}\right) \boldsymbol{\xi}_{i}\right],
\end{aligned}
$$

where $K_{i}>0, \mu_{i}>0$, and $\varepsilon_{i}>0$ are constants. $\pi_{i}$ and $\vartheta_{i}$ are odd positive integers such that $\pi_{i}<\vartheta_{i}$.

Theorem 3. Consider the faulty distributed coordinate spacecraft attitude control system; for each spacecraft, design the following sliding mode-base observer $\widehat{\mathbf{y}}_{i_{-1}}$ to reconstruct the lumped fault $\mathbf{y}_{i_{1}-1}$ :

$$
\widehat{\mathbf{y}}_{i_{-1}}=K_{i} \mathbf{M}_{i}+\mu_{i} \operatorname{sgn}\left(\mathbf{M}_{i}\right)+\varepsilon_{i} \mathbf{M}_{i}^{\pi_{i} / \vartheta_{i}}+\mathbf{C}_{i}\left(\mathbf{x}_{i_{1} 1}, \mathbf{x}_{i_{-}-}\right) \mathbf{M}_{i} .
$$


If the observer gain $\mu_{i}$ is chosen such that

$$
\mu_{i}>8 \gamma_{i}
$$

then $\widehat{\mathbf{y}}_{i_{1} 1}$ will converge to $\mathbf{y}_{i_{1} 1}$ with finite-time convergence, and its reconstruction error $\widetilde{\mathbf{y}}_{i_{-} 1}=\widehat{\mathbf{y}}_{i_{-} 1}-\mathbf{y}_{i_{-} 1}$ will converge to zero in finite time.

Proof. Choose a Lyapunov candidate function as $V_{i}=$ $(1 / 2) \mathbf{M}_{i}^{\mathrm{T}} \overline{\mathbf{J}}_{i}\left(\mathbf{x}_{i_{1} 1}\right) \mathbf{M}_{i}$. Then, differentiating both sides of $V_{i}$, it leads to

$$
\begin{aligned}
\dot{V}_{i} & =\frac{1}{2} \mathbf{M}_{i}^{\mathrm{T}} \dot{\overline{\mathbf{J}}}_{i}\left(\mathbf{x}_{i_{-} 1}\right) \dot{\mathbf{M}}_{i}+\mathbf{M}_{i}^{\mathrm{T}} \overline{\mathbf{J}}_{i}\left(\mathbf{x}_{i_{-} 1}\right) \dot{\mathbf{M}}_{i} \\
& =\frac{1}{2} \mathbf{M}_{i}^{\mathrm{T}} \dot{\overline{\mathbf{J}}}_{i}\left(\mathbf{x}_{i_{-} 1}\right) \dot{\mathbf{M}}_{i}+\mathbf{M}_{i}^{\mathrm{T}} \overline{\mathbf{J}}_{i}\left(\mathbf{x}_{i_{-} 1}\right)\left(\dot{\mathbf{x}}_{i_{2}}-\dot{\boldsymbol{\xi}}_{i}\right) .
\end{aligned}
$$

Using (27) and Property 3, inserting (24) into (31) yields

$$
\begin{aligned}
\dot{V}_{i}= & -\mathbf{M}_{i}^{\mathrm{T}}\left[K_{i} \mathbf{M}_{i}+\mu_{i} \mathbf{s g n}\left(\mathbf{M}_{i}\right)+\varepsilon_{i} \mathbf{M}_{i}^{\pi_{i} / \vartheta_{i}}\right. \\
& \left.+\left(\mathbf{T}_{i}\left(\boldsymbol{\sigma}_{b}^{i}\right)\right)^{\mathrm{T}} \mathbf{u}_{\mathrm{nor} i}-\mathbf{C}_{i}\left(\mathbf{x}_{i_{-1}}, \mathbf{x}_{i_{-} 2}\right) \boldsymbol{\xi}_{i}\right] \\
& +\frac{1}{2} \mathbf{M}_{i}^{\mathrm{T}} \dot{\overline{\mathbf{J}}}_{i}\left(\mathbf{x}_{i_{-} 1}\right) \mathbf{M}_{i} \\
& +\mathbf{M}_{i}^{\mathrm{T}}\left[-\mathbf{C}_{i}\left(\mathbf{x}_{i_{-1} 1}, \mathbf{x}_{i_{-2}}\right) \mathbf{x}_{i \_2}+\left(\mathbf{T}_{i}\left(\boldsymbol{\sigma}_{b}^{i}\right)\right)^{\mathrm{T}} \mathbf{u}_{\mathrm{nor} i}+\mathbf{y}_{i_{-} 1}\right] \\
= & -K_{i} \mathbf{M}_{i}^{\mathrm{T}} \mathbf{M}_{i}-\mu_{i} \mathbf{M}_{i}^{\mathrm{T}} \mathbf{s g n}\left(\mathbf{M}_{i}\right) \\
& -\varepsilon_{i} \mathbf{M}_{i}^{\left(\pi_{i}+\vartheta_{i}\right) / \vartheta_{i}}+\mathbf{M}_{i}^{\mathrm{T}} \mathbf{y}_{i_{-1} 1} .
\end{aligned}
$$

From Theorem 1, it is known that $\left\|\left(\mathbf{T}_{i}\left(\boldsymbol{\sigma}_{b}^{i}\right)\right)^{\mathrm{T}} \mathbf{u}_{\text {fault } i}\right\| \leq 8 \gamma_{i}$; that is, $\left\|\mathbf{y}_{i_{1} 1}\right\| \leq 8 \gamma_{i}$. Then, with the choice of the observer gain in (30), it leaves (32) as

$$
\begin{aligned}
\dot{V}_{i} & \leq-K_{i} \mathbf{M}_{i}^{\mathrm{T}} \mathbf{M}_{i}-\varepsilon_{i} \mathbf{M}_{i}^{\left(\pi_{i}+\vartheta_{i}\right) / \vartheta_{i}} \\
& \leq-2 K_{i} V_{i}-\varepsilon_{i} 2^{\left(\pi_{i}+\vartheta_{i}\right) / 2 \vartheta_{i}}\left(V_{i}\right)^{\left(\pi_{i}+\vartheta_{i}\right) / 2 \vartheta_{i}}
\end{aligned}
$$

Consequently, using Lemma 2, it can be obtained from (33) that $V_{i}(t) \equiv 0$ for all $t \geq t_{f_{i}}$ :

$$
\begin{aligned}
t_{f} \leq & \frac{1}{2 K_{i}\left(1+\left(\pi_{i}+\vartheta_{i}\right) / 2 \vartheta_{i}\right)} \\
& \cdot \ln \frac{2 K_{i} V_{i}^{\left(\vartheta_{i}-\pi_{i}\right) / 2 \vartheta_{i}}(0)+\varepsilon_{i} 2^{\left(\pi_{i}+\vartheta_{i}\right) / 2 \vartheta_{i}}}{\varepsilon_{i} 2^{\left(\pi_{i}+\vartheta_{i}\right) / 2 \vartheta_{i}}} .
\end{aligned}
$$

At this time, applying the definition of $V_{i}$, one has $\mathbf{M}_{i}(t) \equiv \mathbf{0}$ for all $t \geq t_{f_{i}}$. Therefore, for all $t \geq t_{f_{-} i}$, it leads to

$$
\dot{\mathbf{M}}_{i}(t) \equiv \mathbf{0}
$$

On the other hand, it follows from the reconstruction error $\widetilde{\mathbf{y}}_{i_{-} 1}$ that

$$
\begin{aligned}
\widetilde{\mathbf{y}}_{i_{-} 1}= & \widehat{\mathbf{y}}_{i_{-} 1}-\mathbf{y}_{i_{-} 1} \\
= & K_{i} \mathbf{M}_{i}+\mu_{i} \mathbf{s g n}\left(\mathbf{M}_{i}\right)+\varepsilon_{i} \mathbf{M}_{i}^{\pi_{i} / \vartheta_{i}} \\
& +\mathbf{C}_{i}\left(\mathbf{x}_{i_{-} 1}, \mathbf{x}_{i_{-} 2}\right) \mathbf{M}_{i}-\overline{\mathbf{J}}_{i}\left(\mathbf{x}_{i_{-} 1}\right) \dot{\mathbf{x}}_{i_{-} 2} \\
& +\left(\mathbf{T}_{i}\left(\boldsymbol{\sigma}_{b}^{i}\right)\right)^{\mathrm{T}} \mathbf{u}_{\text {nor } i}-\mathbf{C}_{i}\left(\mathbf{x}_{i_{-} 1}, \mathbf{x}_{i_{-} 2}\right) \mathbf{x}_{i_{-} 2} \\
= & -\overline{\mathbf{J}}_{i}\left(\mathbf{x}_{i_{-} 1}\right)\left[\dot{\mathbf{x}}_{i_{-} 2}-\overline{\mathbf{J}}_{i}^{-1}\left(\mathbf{x}_{i_{-} 1}\right)\right. \\
& \quad \cdot\left(K_{i} \mathbf{M}_{i}+\mu_{i} \mathbf{s g n}\left(\mathbf{M}_{i}\right)+\varepsilon_{i} \mathbf{M}_{i}^{\pi_{i} / \vartheta_{i}}\right. \\
& \left.\left.+\left(\mathbf{T}_{i}\left(\boldsymbol{\sigma}_{b}^{i}\right)\right)^{\mathrm{T}} \mathbf{u}_{\text {nor } i}-\mathbf{C}_{i}\left(\mathbf{x}_{i_{-1} 1}, \mathbf{x}_{i \_2}\right) \boldsymbol{\xi}_{i}\right)\right] \\
= & -\overline{\mathbf{J}}_{i}^{-1}\left(\mathbf{x}_{i_{-} 1}\right) \dot{\mathbf{M}}_{i} .
\end{aligned}
$$

As a result, it is obtained from (35) and (36) that $\widetilde{\mathbf{y}}_{i_{-} 1}(t) \equiv 0$ for all $t \geq t_{f i}$. From this, we can conclude that the lumped fault reconstruction error $\widetilde{\mathbf{y}}_{i_{1} 1}(t)$ of the proposed sliding mode observer converges to zero within finite time. Thereby the proof is completed here.

Theorem 4. For all possible thruster faults in each spacecraft of the considered distributed coordinated attitude system, the fault $\mathbf{u}_{\text {fault } i}$ occurring in the ith spacecraft can be reconstructed by the signal $\left(\mathbf{G}_{i}\left(\boldsymbol{\sigma}_{b}^{i}\right)\right)^{\mathrm{T}} \widehat{\mathbf{y}}_{i_{-1}}$ in finite-time $t_{f_{-} i}$. That is, $\mathbf{u}_{\text {fault } i}(t) \equiv$ $\left(\mathbf{G}_{i}\left(\boldsymbol{\sigma}_{b}^{i}\right)\right)^{\mathrm{T}} \widehat{\mathbf{y}}_{i_{-} 1}$ for all $t \geq t_{f i}$.

Proof. It follows from (22) that

$$
\mathbf{u}_{\text {fault } i}=\left(\mathbf{G}_{i}\left(\boldsymbol{\sigma}_{b}^{i}\right)\right)^{\mathrm{T}}\left(\widehat{\mathbf{y}}_{i_{-} 1}-\widetilde{\mathbf{y}}_{i_{-} 1}\right) .
$$

As stated in Theorem 3 , it is seen that $\widehat{\mathbf{y}}_{i_{1} 1}$ will converge to $\mathbf{y}_{i_{1} 1}$ with finite-time convergence. More specifically, it has $\widetilde{\mathbf{y}}_{i_{-} 1}(t) \equiv 0$ for all $t \geq t_{f_{-} i}$. Hence, it leaves (37)

$$
\mathbf{u}_{\text {fault } \_i}(t) \equiv\left(\mathbf{G}_{i}\left(\boldsymbol{\sigma}_{b}^{i}\right)\right)^{\mathrm{T}} \widehat{\mathbf{y}}_{i_{-} 1}, \quad t \geq t_{f_{-i}},
$$

which implies that $\mathbf{u}_{\text {fault } i}$ can be reconstructed by $\left(\mathbf{G}_{i}\left(\boldsymbol{\sigma}_{b}^{i}\right)\right)^{\mathrm{T}} \widehat{\mathbf{y}}_{i_{-} 1}$ with zero reconstruction error in a finite-time $t_{f i}$. If one defines the reconstruction error between $\mathbf{u}_{\text {fault } i}$ and $\left(\mathbf{G}_{i}\left(\boldsymbol{\sigma}_{b}^{i}\right)\right)^{\mathrm{T}} \widehat{\mathbf{y}}_{i_{-} 1}$ as $\mathbf{e}_{i}(t)=\left[\begin{array}{lll}e_{i 1} & e_{i 2} & e_{i 3}\end{array}\right]^{\mathrm{T}}$, then it has $\mathbf{e}_{i}(t) \equiv$ 0 for all $t \geq t_{f i}$. Hence, the proof of Theorem 3 is completed.

\section{Simulation Results}

To illustrate the performance of the proposed fault reconstruction approach, three spacecrafts in a distributed coordinate configuration are considered in the simulation; that is, $n=3$. Those spacecrafts' inertia are $\mathbf{J}_{1}=$ $\operatorname{diag}\left(\left[\begin{array}{lll}20 & 17 & 15\end{array}\right]^{\mathrm{T}}\right), \mathbf{J}_{2}=\operatorname{diag}\left(\left[\begin{array}{lll}22.5 & 25 & 27.5\end{array}\right]^{\mathrm{T}}\right)$, and $\mathbf{J}_{3}=$ $\operatorname{diag}\left(\left[\begin{array}{lll}27.5 & 30 & 25.5\end{array}\right]^{\mathrm{T}}\right)$, respectively. Each spacecraft mounts 
TABLE 1: Thruster's layout $(i=1,2, \ldots, n$ and $j=1,2, \ldots, 12)$.

\begin{tabular}{lccccc}
\hline $\begin{array}{l}\text { Thruster } \\
\text { number }\end{array}$ & \multicolumn{2}{c}{$\begin{array}{c}\text { Position in } \mathscr{F}_{b}^{i} \text { of the } \\
\text { attitude control system }\end{array}$} & \multicolumn{2}{c}{ Orientation in $\mathscr{F}_{b}^{i}$} \\
& $r_{x i j}^{0}(\mathrm{~mm})$ & $r_{y i j}^{0}(\mathrm{~mm})$ & $r_{z i j}^{0}(\mathrm{~mm})$ & $\alpha_{i j}^{0}(\mathrm{deg})$ & $\beta_{i j}^{0}(\mathrm{deg})$ \\
\hline$\# \mathrm{~T}_{1}$ & 700 & 0 & 0 & 0 & -90 \\
$\# \mathrm{~T}_{2}$ & 700 & 0 & 0 & 0 & 90 \\
$\# \mathrm{~T}_{3}$ & -700 & 0 & 0 & 0 & -90 \\
$\# \mathrm{~T}_{4}$ & -700 & 0 & 0 & 0 & 90 \\
$\# \mathrm{~T}_{5}$ & 0 & 800 & 0 & -90 & 90 \\
$\# \mathrm{~T}_{6}$ & 0 & 800 & 0 & 90 & 90 \\
$\# \mathrm{~T}_{7}$ & 0 & -800 & 0 & -90 & 90 \\
$\# \mathrm{~T}_{8}$ & 0 & -800 & 0 & 90 & 90 \\
$\# \mathrm{~T}_{9}$ & 0 & 0 & -700 & 0 & 0 \\
$\# \mathrm{~T}_{10}$ & 0 & 0 & -700 & 0 & 180 \\
$\# \mathrm{~T}_{11}$ & 0 & 0 & 700 & 0 & 0 \\
$\# \mathrm{~T}_{12}$ & 0 & 0 & 700 & 0 & 180 \\
\hline
\end{tabular}

twelve thrusters to perform large-angle attitude maneuver; that is, $N_{i}=12, i=1,2, \ldots, n$. For the $i$ th spacecraft, those twelve thrusters are assumed to be distributed symmetrically on three axes of $\mathscr{F}_{b}^{i}$, and the propulsion force is perpendicular to the corresponding axis such that the distribution matrix can be simply determined by the distance $\mathbf{r}_{i j}^{0}, j=1,2, \ldots, 12$. The mechanical configuration of thrusters is illustrated in Figure 3 . The thrusters are commanded in pulse delay and duration, providing a nominal force $F$ of $0.8 \mathrm{~N}$. The nominal positions (i.e., the point of application of the main thrust force) and orientation (azimuth and elevation) of thrusters are reported in Table 1.

In simulation, the initial conditions of the $i$ th spacecraft with the quaternion and the angular velocity are $\sigma_{b}^{1}(0)=$ $\left[\begin{array}{lll}0.288 & -0.143 & -0.020\end{array}\right]^{\mathrm{T}}, \boldsymbol{\sigma}_{b}^{2}(0)=\left[\begin{array}{lll}0.4 & 0.3 & -0.15\end{array}\right]^{\mathrm{T}}, \boldsymbol{\sigma}_{b}^{3}(0)=$ $\left[\begin{array}{lll}-0.65 & 0.23 & 0.45\end{array}\right]^{\mathrm{T}}$, and $\boldsymbol{\omega}_{b}^{i}(0)=\left[\begin{array}{lll}0 & 0 & 0\end{array}\right]^{\mathrm{T}} \mathrm{rad} / \mathrm{sec}$, respectively. The parameters for the proposed reconstruction scheme are chosen as $K_{i}=0.75, \varepsilon_{i}=1.5, \mu_{i}=14.5, \pi_{i}=13$, and $\vartheta_{i}=19, i=1,2, \ldots, n$.

4.1. Thruster Faults. To investigate the fault reconstruction performance of the proposed scheme, the following thrust magnitude error scenarios and thruster's misalignments are introduced and simulated.

(i) A random misalignment is added each time the thruster is switched on, which corresponds to a random thrust misalignment. The model for this misalignment is a Gaussian noise on both the azimuth and the elevation angles (i.e., $\Delta \alpha_{i j}$ and $\Delta \beta_{i j}$, resp.; $i=1,2, \ldots, n$ and $j=1,2, \ldots, 12)$, each with standard deviation of 2.5\% (Figure 1).

(ii) Supplementary position of thruster $\Delta \mathbf{r}_{i j}, i=1,2$, $\ldots, n, j=1,2, \ldots, 12$, is modeled as a Gaussian noise, each with standard deviation of $4 \%$.

(iii) For all the twelve thrusters in each spacecraft, the force delivered is modeled with a nominal value signal

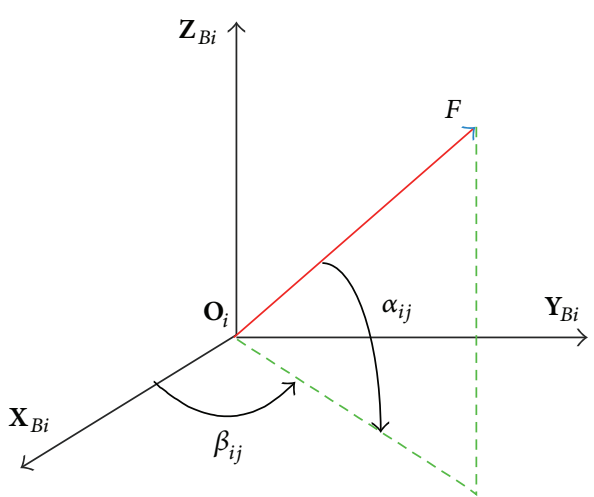

FIgURE 1: Azimuth and elevation of the $j$ th thruster in the $i$ th spacecraft.

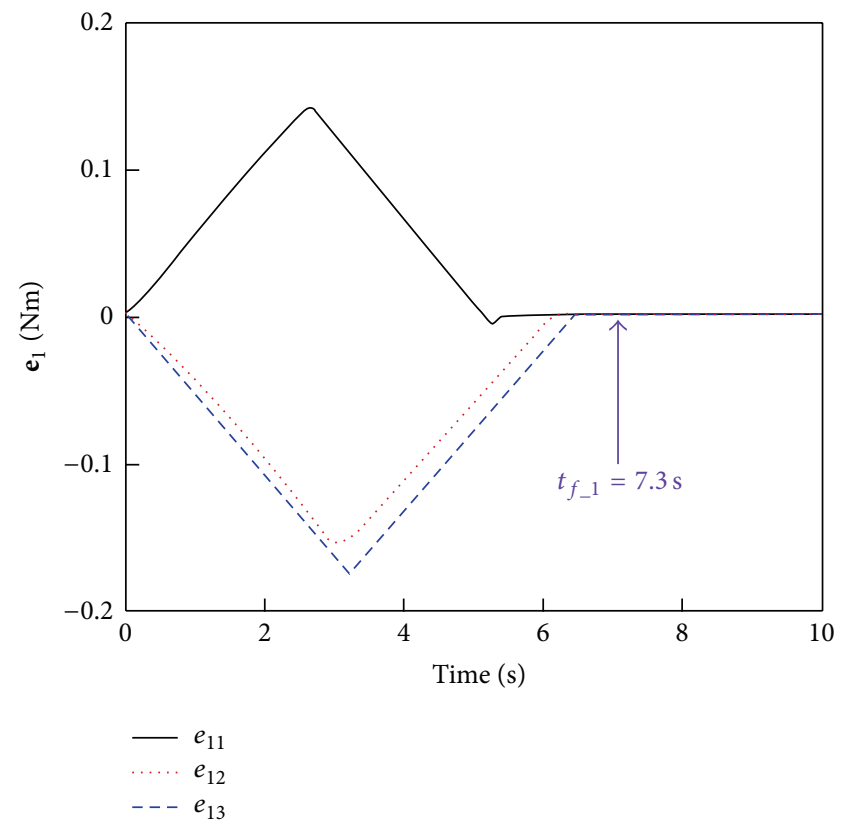

FIgURE 2: The reconstruction error $\mathbf{e}_{1}$.

of $F=0.8 \mathrm{~N}$ corrupted by a zero-mean Gaussian noise with a constant standard deviation of $0.35 \mathrm{~N}$.

4.2. Control Performance. When the proposed reconstruction approach for thruster faults is applied to each spacecraft of the distributed coordinate attitude system, the sliding mode observer-based reconstruction scheme leads to a good reconstruction performance. Figure 2 illustrates the error between $\mathbf{u}_{\text {fault_1 }}$ and its reconstruction value $\left(\mathbf{G}_{1}\left(\boldsymbol{\sigma}_{b}^{1}\right)\right)^{\mathrm{T}} \widehat{\mathbf{y}}_{1_{1} 1}$. It is seen that the reconstruction error $\mathbf{e}_{1}(t)$ converges to zero in a finite-time $t_{f_{-1}}=7.3$ seconds. For the 2 nd spacecraft, it is obtained from the reconstruction approach that the reconstruction error $\mathbf{e}_{2}(t)$ is finite-time stable, and its converging time is $t_{f 2}=17.5$ seconds, as we can see in Figure 3. Moreover, it is shown in Figure 4 that the reconstruction approach is able to reconstruct the faults occurring in the third spacecraft. This reconstruction is accomplished 


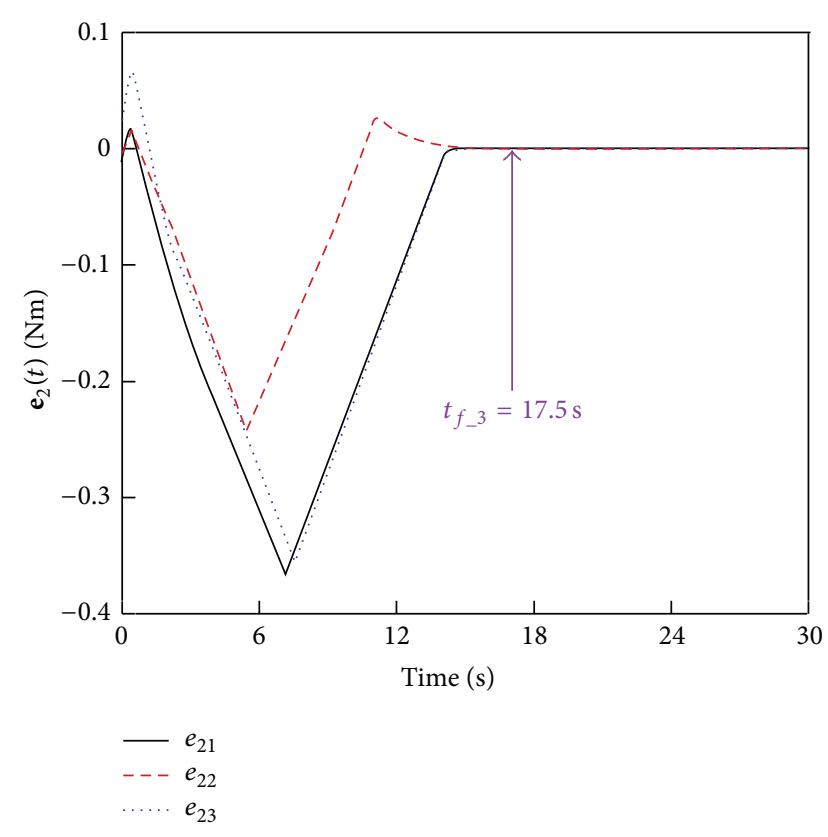

FIGURE 3: The reconstruction error $\mathbf{e}_{2}$.

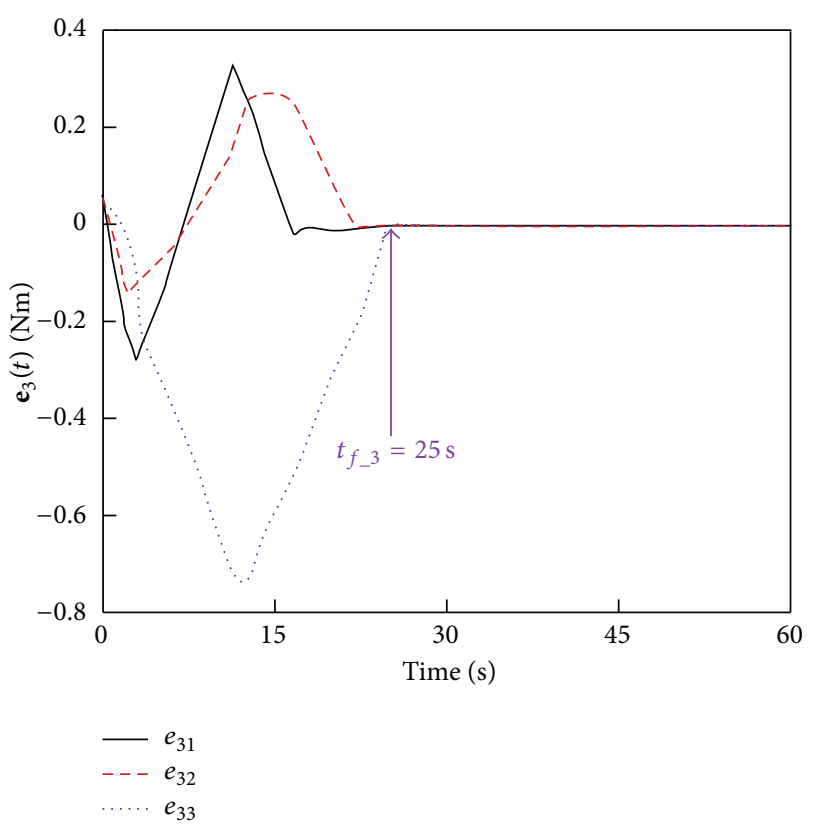

FIgURE 4: The reconstruction error $\mathbf{e}_{3}$ for the third spacecraft.

in a finite-time $t_{f_{-} 3}=25$ seconds. From those results, it is seen that a faster reconstruction of the thruster faults in the considered distributed coordinate spacecraft attitude control system is achieved.

\section{Conclusions and Future Work}

A sliding mode observer-based fault reconstruction scheme was proposed for each agent in a distributed coordinated spacecraft attitude control system. Thruster faults were investigated. The proposed reconstruction approach guaranteed that all possible thruster faults were reconstructed with zero reconstruction error. Moreover, it was able to accomplish a faster fault reconstruction, because it was capable of making the reconstructed signal converge to the actual fault within finite time. By choosing the observer gains, such finite time can be tuned by the designer. If the spacecraft was only under the effect of external disturbance, then this approach can also reconstruct the magnitude of external disturbance with finite-time convergence. This was another feature of this approach. However, this work only carried out fault reconstruction for distributed coordinate spacecraft system; the attitude controller design was not done. As some of future works, reliable attitude controller should be carried out by using the reconstructed fault signals obtained from the reconstruction approach in this work.

\section{Conflict of Interests}

The authors declare that there is no conflict of interests regarding the publication of this paper.

\section{References}

[1] R. Burns, C. A. McLaughlin, J. Leitner, and M. Martin, "TechSat 21: Formation design, control, and simulation," in Proceedings of the IEEE Aerospace Conference, pp. 19-25, March 2000.

[2] H. J. Yang, X. You, Y. Q. Xiu, and H. B. Li, "Adaptive control for attitude synchronisation of spacecraft formation via extended state observer," IET Control Theory \& Applications, vol. 8, no. 18, pp. 2171-2185, 2014.

[3] L. Zhao and Y. M. Jia, "Decentralized adaptive attitude synchronization control for spacecraft formation using nonsingular fast terminal sliding mode," Nonlinear Dynamics, vol. 78, no. 4, pp. 2779-2794, 2014.

[4] H. J. Yang, X. You, Y. Q. Xia, and Z. X. Liu, "Nonlinear attitude tracking control for spacecraft formation with multiple delays," Advances in Space Research, vol. 54, pp. 759-769, 2014.

[5] R. Kristiansen, E. I. Grøtli, P. J. Nicklasson, and J. T. Gravdahl, "A model of relative translation and rotation in leader-follower spacecraft formations," Modeling, Identification and Control, vol. 28, no. 1, pp. 3-13, 2007.

[6] J. Li and K. D. Kumar, "Decentralized fault-tolerant control for satellite attitude synchronization," IEEE Transactions on Fuzzy Systems, vol. 20, no. 3, pp. 572-586, 2012.

[7] B. Wu, D. Wang, and E. K. Poh, "Decentralized sliding-mode control for attitude synchronization in spacecraft formation," International Journal of Robust and Nonlinear Control, vol. 23, no. 11, pp. 1183-1197, 2013.

[8] B.-L. Cong, X.-D. Liu, and Z. Chen, "Distributed attitude synchronization of formation flying via consensus-based virtual structure," Acta Astronautica, vol. 68, no. 11-12, pp. 1973-1986, 2011.

[9] R. W. Beard, J. Lawton, and F. Y. Hadaegh, "A coordination architecture for spacecraft formation control," IEEE Transactions on Control Systems Technology, vol. 9, no. 6, pp. 777-790, 2001. 
[10] H. Z. Liang, J. Y. Wang, and Z. W. Sun, "Robust decentralized coordinated attitude control of spacecraft formation," Acta Astronautica, vol. 69, no. 5-6, pp. 280-288, 2011.

[11] J. Zhou, Q. Hu, and M. I. Friswell, "Decentralized finite time attitude synchronization control of satellite formation flying," Journal of Guidance, Control, and Dynamics, vol. 36, no. 1, pp. 185-195, 2013.

[12] M. Tafazoli, "A study of on-orbit spacecraft failures," Acta Astronautica, vol. 64, no. 2-3, pp. 195-205, 2009.

[13] Y. W. Liang, S. D. Xu, and C. L. Tsai, "Study of VSC reliable designs with application to spacecraft attitude stabilization," IEEE Transactions on Control Systems Technology, vol. 15, no. 2, pp. 332-338, 2007.

[14] W. C. Cai, X. H. Liao, and Y. D. Song, "Indirect robust adaptive fault-tolerant control for attitude tracking of spacecraft," Journal of Guidance, Control, and Dynamics, vol. 31, no. 5, pp. 1456-1463, 2008.

[15] S. Varma and K. D. Kumar, "Fault tolerant satellite attitude control using solar radiation pressure based on nonlinear adaptive sliding mode," Acta Astronautica, vol. 66, no. 3-4, pp. 486500,2010

[16] Godard and K. D. Kumar, "Fault tolerant reconfigurable satellite formations using adaptive variable structure techniques," Journal of Guidance, Control, and Dynamics, vol. 33, no. 3, pp. 969984, 2010.

[17] B. Xiao, Q. L. Hu, and Y. M. Zhang, "Adaptive sliding mode fault tolerant attitude tracking control for flexible spacecraft under actuator saturation," IEEE Transactions on Control Systems Technology, vol. 20, no. 6, pp. 1605-1612, 2012.

[18] R. Muradore and P. Fiorini, "A PLS-based statistical approach for fault detection and isolation of robotic manipulators," IEEE Transactions on Industrial Electronics, vol. 59, no. 8, pp. 31673175, 2012.

[19] X.-G. Yan and C. Edwards, "Adaptive sliding-mode-observerbased fault reconstruction for nonlinear systems with parametric uncertainties," IEEE Transactions on Industrial Electronics, vol. 55, no. 11, pp. 4029-4036, 2008.

[20] S. Yin, S. Ding, X. Xie, and H. Huo, "A review on basic datadriven approaches for industrial process monitoring," IEEE Transactions on Industrial Electronics, vol. 61, no. 11, pp. 64186428, 2014.

[21] S. Yin, X. Li, H. Gao, and O. Kaynak, "Data-based techniques focused on modern industry: an 14 overview," IEEE Transactions on Industrial Electronics, vol. 62, no. 1, pp. 657-667, 2015.

[22] Z. Q. Li, L. Ma, and K. Khorasani, "A dynamic neural networkbased reaction wheel fault diagnosis for satellites," in Proceedings of the International Joint Conference on Neural Networks (IJCNN '06), pp. 3714-3721, Vancouver, BC, Canada, July 2006.

[23] T. Jiang and K. Khorasani, "A fault detection, isolation and reconstruction strategy for a satellite's attitude control subsystem with redundant reaction wheels," in Proceedings of the IEEE International Conference on Systems, Man, and Cybernetics (SMC '07), pp. 3146-3152, Montreal, Canada, October 2007.

[24] B. Chen and S. Nagarajaiah, "Linear-matrix-inequality-based robust fault detection and isolation using the eigenstructure assignment method," Journal of Guidance, Control, and Dynamics, vol. 30, no. 6, pp. 1831-1835, 2007.

[25] Q. Hou, Y. H. Cheng, N. Y. Lu, and B. Jiang, "Study on FDD and FTC of satellite attitude control system based on the effectiveness factor," in Proceedings of the 2nd International Symposium on Systems and Control in Aerospace and Astronautics (ISSCAA '08), pp. 1096-1101, Shenzhen, China, December 2008.
[26] D. Henry, "Fault diagnosis of microscope satellite thrusters using $H_{\infty} / H_{-}$filters," Journal of Guidance, Control, and Dynamics, vol. 31, no. 3, pp. 699-711, 2008.

[27] W. R. Williamson, J. L. Speyer, V. T. Dang, and J. Sharp, "Fault detection and isolation for deep space satellites," Journal of Guidance, Control, and Dynamics, vol. 32, no. 5, pp. 1570-1584, 2009.

[28] R. J. Patton, F. J. Uppal, S. Simani, and B. Polle, "Robust FDI applied to thruster faults of a satellite system," Control Engineering Practice, vol. 18, no. 9, pp. 1093-1109, 2010.

[29] I. Nagesh and C. Edwards, "A sliding mode observer based FDI scheme for a nonlinear satellite systems," in Proceedings of the 20th IEEE International Conference on Control Applications (CCA '11), pp. 159-164, Denver, Colo, USA, September 2011.

[30] I. Nagesh and C. Edwards, "A sliding mode observer based sensor fault detection and isolation scheme for a nonlinear satellite system," in Proceedings of the 12th International Workshop on Variable Structure Systems (VSS '12), pp. 248-253, Mumbai, India, January 2012.

[31] H. Yoon and P. Tsiotras, "Adaptive spacecraft attitude tracking control with actuator uncertainties," in Proceedings of the AIAA Guidance, Navigation, and Control Conference, pp. 6392-6404, San Francisco, Calif, USA, August 2005.

[32] S. K. Scarritt, "Nonlinear model reference adaptive control for satellite attitude tracking," in Proceedings of the AIAA Guidance, Navigatoin and Control Conference and Exhibit, Honolulu, Hawaii, USA, August 2008.

[33] A. M. Fosbury and C. K. Nebelecky, "Spacecraft actuator alignment estimation," in Proceedings of the AIAA Guidance, Navigation, and Control Conference, Chicago, Ill, USA, August 2009.

[34] H.-C. Lim and H. Bang, "Adaptive control for satellite formation flying under thrust misalignment," Acta Astronautica, vol. 65, no. 1-2, pp. 112-122, 2009.

[35] J. L. Crassidis and F. L. Markley, "Sliding mode control using modified Rodrigues parameters," Journal of Guidance, Control, and Dynamics, vol. 19, no. 6, pp. 1381-1383, 1996.

[36] M. J. Sidi, Spacecraft Dynamics and Control, Cambridge University Press, Cambridge, Mass, USA, 1997.

[37] A. Valdes and K. Khorasani, "A pulsed plasma thruster fault detection and isolation strategy for formation flying of satellites," Applied Soft Computing Journal, vol. 10, no. 3, pp. 746-758, 2010.

[38] B. Xiao, Q. Hu, W. Singhose, and X. Huo, "Reaction wheel fault compensation and disturbance rejection for spacecraft attitude tracking," Journal of Guidance, Control, and Dynamics, vol. 36, no. 6, pp. 1565-1575, 2013.

[39] M. Chen, Q.-X. Wu, and R.-X. Cui, "Terminal sliding mode tracking control for a class of SISO uncertain nonlinear systems," ISA Transactions, vol. 52, no. 2, pp. 198-206, 2013. 


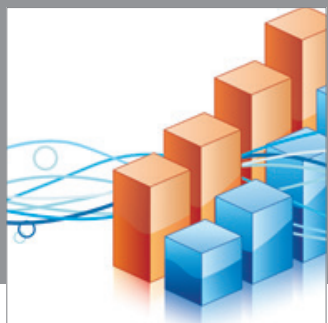

Advances in

Operations Research

mansans

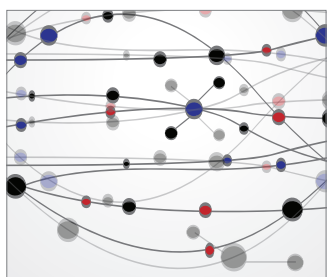

The Scientific World Journal
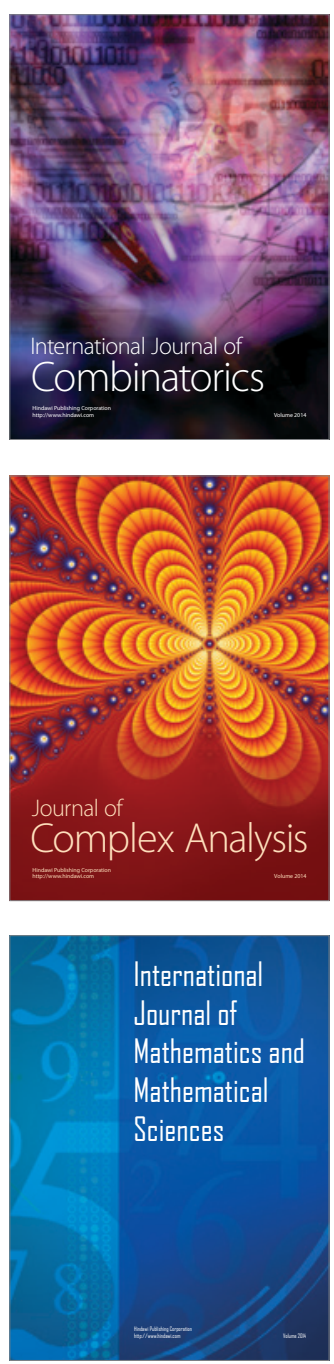
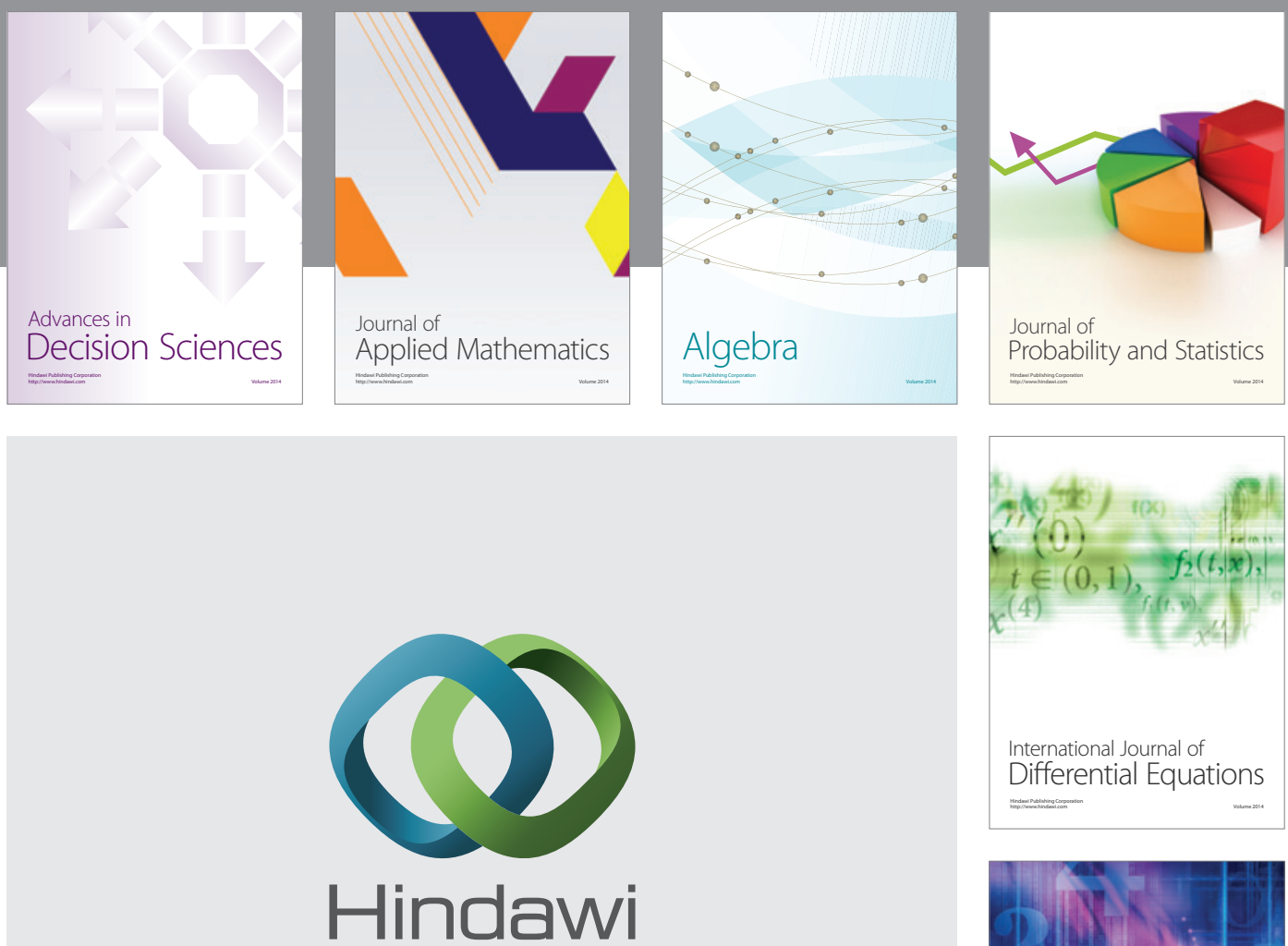

Submit your manuscripts at http://www.hindawi.com
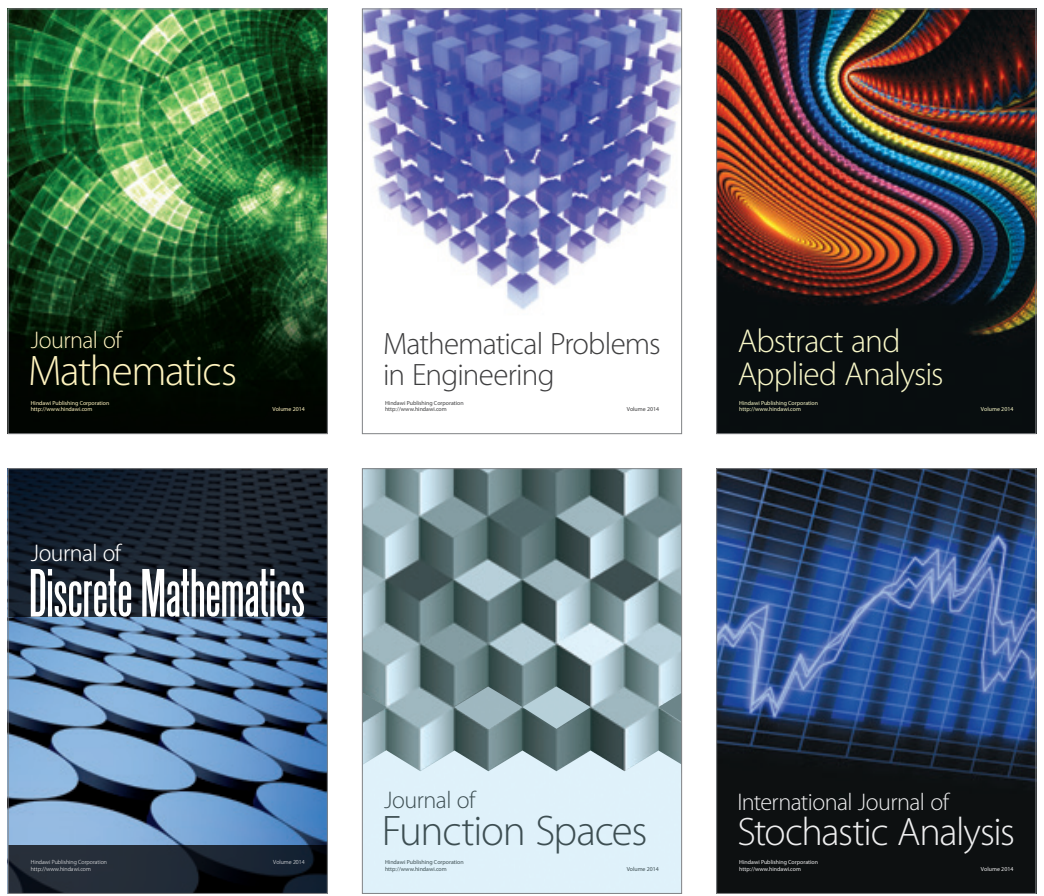

Journal of

Function Spaces

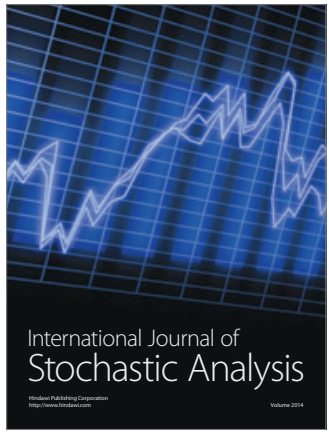

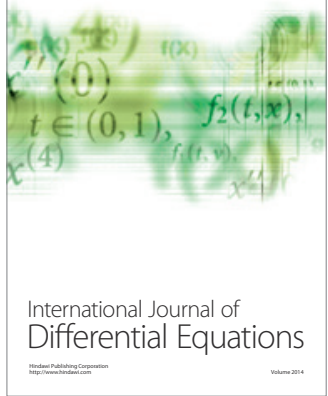
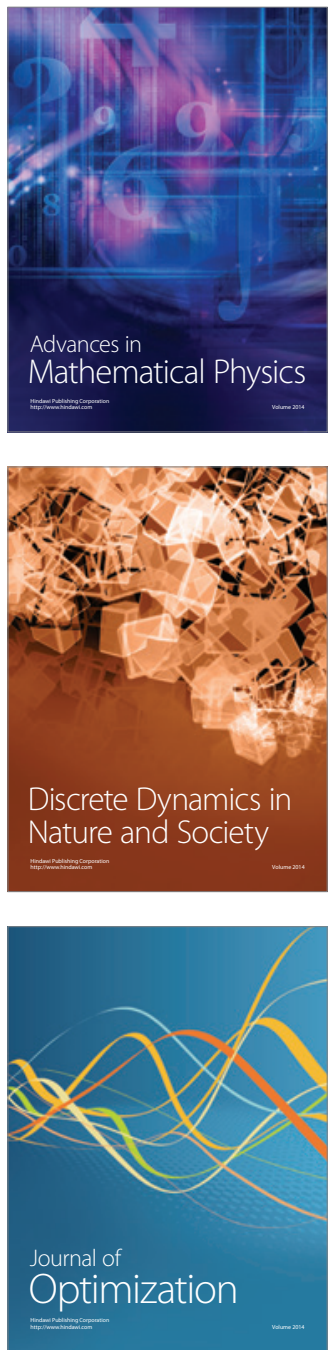